einer starken parlamentarischen corporate identity, was durchaus auch in den Strukturen des parlamentarischen Regierungssystems möglich wäre und zu einer Re-Parlamentarisierung beitragen könnte.

So kann zusammenfassend der Schluss gezogen werden, dass eine Diskrepanz besteht zwischen den Rechtstexten und der politischen Wirklichkeit - nicht neu in Ungarn. Ursächlich sind sowohl Grundparameter des politischen Systems als auch Faktoren der Politischen Kultur. Die Verbindung beider erklärt bestens die Parallelität von Wandel und Beharrung des ungarischen Parlamentarismus vor dem Hintergrund der Teilnahme des Landes an der Europäischen Integration. Eine Veränderung hin zur politischen Stärkung des Parlaments könnten lediglich eine Abnahme der parteipolitischen Durchdringung der Elite beziehungsweise eine Minderheitsregierung initiieren. Beide Varianten sind eher unwahrscheinlich.

\title{
Vertikale Gewaltenteilung: institutionenpolitische Leitidee oder demokratietheoretische Chiffre? Reform westeuropäischer Bundes- und Einheitsstaaten im Vergleich
}

\author{
Florian Grotz
}

Föderalismus als Organisationsprinzip politischer Herrschaft erfreut sich derzeit großer Beliebtheit. ${ }^{1}$ Zwar ist die überwiegende Staatenmehrheit noch immer unitarisch verfasst, doch gibt es eine Reihe von Ländern, die in der jüngeren Vergangenheit eine bundesstaatliche Ordnung eingeführt haben, zum Beispiel Äthiopien, Belgien, Bosnien-Herzegowina oder Südafrika. Hinzu tritt eine weit höhere Zahl von Staaten, die eine Abkehr vom unitarischen Prinzip vollzogen haben oder zumindest anstreben. Dies gilt auch und gerade für Westeuropa. Dort wurden nicht nur die größeren Einheitsstaaten seit Ende der 1970er Jahre einer substanziellen Dezentralisierung unterzogen (Frankreich, Großbritannien, Italien, Spanien); vielmehr fanden sich auch in den unitarischen Bundesstaaten des Kontinents Reformansätze, die als Versuche zur „(Wieder-)Belebung“ des Föderalismus gelten können (Deutschland, Österreich).

Fragt man nach den Gründen für diese länderübergreifenden Reformtendenzen, fällt zunächst ein Argument ins Auge, das sich schon bei den „Klassikern“ der Vergleichenden Regierungslehre findet: Demnach erscheint das Föderalismusprinzip für die Entwicklung der Demokratie insofern förderlich, als eine vertikale Aufteilung hoheitlicher Kompetenzen die Machtposition des Zentralstaates einschränkt und damit der Mehrheitsherrschaft inner-

1 Der vorliegende Beitrag bildet die erweiterte und aktualisierte Fassung eines Vortrags, der im Rahmen des Workshops „Föderale Gewaltenteilung und Demokratie“ der Sektion „Vergleichende Politikwissenschaft" der Deutschen Vereinigung für Politische Wissenschaft (DVPW) am 27. September 2006 gehalten wurde. Für hilfreiche Kommentare und Anregungen danke ich den Diskussionsteilnehmern sowie den Mitgliedern der ZParl-Redaktion. 
halb des nationalen Regierungssystems Grenzen setzt. ${ }^{2}$ Auch in der neueren Föderalismusdiskussion ist diese Position prominent vertreten. ${ }^{3}$ Nicht zuletzt hat Arend Lijphart die vertikale Staatsstruktur in seine empirische Demokratietheorie einbezogen ${ }^{4}$, nach der machtteilige Institutionen vor allem für fragmentierte Gesellschaften vorteilhafter sind als machtkonzentrierende Arrangements. ${ }^{5}$

Blickt man jedoch auf die Empirie institutioneller Reformen in etablierten Demokratien, muss - so die These der folgenden Ausführungen - die Bedeutung des Gewaltenteilungsarguments in zweifacher Hinsicht relativiert werden. Zum einen finden sich bezüglich des Verhältnisses von Staatsorganisation ${ }^{6}$ und Demokratie alternative Zielvorstellungen, die mit der Idee der „vertikalen Gewaltenteilung“ konkurrieren und gleichfalls handlungsleitend werden können. Zum anderen bedarf es einer Kontextualisierung solcher Zielvorstellungen insofern, als deren Bedeutung innerhalb eines Reformprozesses von den jeweiligen (macht-)politischen Rahmenbedingungen abhängt.

Der vorliegende Beitrag sucht den Stellenwert der vertikalen Gewaltenteilung als institutionenpolitische Leitidee am Beispiel von Staatsreformen in westeuropäischen Ländern systematisch herauszuarbeiten. Dazu bezieht die Analyse sowohl bundes- als auch einheitsstaatliche Fälle ein, um zu prüfen, ob und inwieweit die benannte These auf beide Grundtypen der Staatsorganisation anwendbar ist. Der folgende Abschnitt befasst sich zunächst allgemein mit den Strukturmerkmalen und Entwicklungsbedingungen von föderalen und unitarischen Staaten. Im Zentrum steht dabei die Frage, unter welchen Rahmenbedingungen die Idee der vertikalen Gewaltenteilung eine erwartbar große Bedeutung für die

2 Carl Joachim Friedrich, Trends of Federalism in Theory and Practice, New York 1968; Karl Loewenstein, Verfassungslehre, 2. Auflage, Tübingen 1969, S. 296 ff. Ideengeschichtlich wegweisend für diese Begründung war die Darstellung der amerikanischen Verfassung durch die Federalists (Alexander Hamilton / John Jay / James Madison, The Federalist: A Commentary on the Constitution of the United States, New York 1961 [zuerst 1787/88]). Für eine differenzierte Interpretation der Federalist Papers in diesem Kontext siehe Jonathan A. Rodden, Hamilton's Paradox. The Promise and Peril of Fiscal Federalism, Cambridge 2006, S. 15 ff.

3 Vgl. unter anderem Robert A. Dahl, Federalism and the Democratic Process, in: ders., Democracy, Liberty and Equality, Bergen 1986, S. 114 - 126; Peter C. Ordenshook / Olga Shvetsova, Federalism and Constitutional Design, in: Journal of Democracy, 8. Jg. (1999), S. 27 - 42; Robert Agranoff(Hrsg.), Accommodating Diversity: Asymmetry in Federal States, Baden-Baden 1999; Juan Linz, Democracy, Multinationalism and Federalism, in: Wolfgang Merkel / Andreas Busch (Hrsg.), Demokratie in Ost und West. Für Klaus von Beyme, Frankfurt am Main 1999, S. 302 - 401 und Ugo M. Amoretti / Nancy Bermeo (Hrsg.), Federalism and Territorial Cleavages, Baltimore / London 2004.

4 Arend Lijphart, Patterns of Democracy. Government Forms and Performance in Thirty-Six Countries, New Haven / London 1999, S. 185 ff.

$5 \mathrm{Zu}$ den von Lijphart bevorzugten konsensorientierten Regelungen zählt bekanntlich auch die Verhältniswahl (im Gegensatz zur Mehrheitswahl). Anders hingegen fällt seine Präferenz bei der Organisation der exekutiv-legislativen Beziehungen aus: Hier optiert er für die mehrheitsdemokratische Form des Parlamentarismus - und damit gegen den „gewaltenteiligen“ Präsidentialismus. Vgl. Arend Lijphart, Constitutional Choices for New Democracies, in: Journal of Democracy, 3. Jg. (1991), S. $72-84$.

6 Der Begriff Staatsorganisation wird hier nur auf das vertikale Beziehungsgefüge zwischen zentralstaatlicher Ebene und subnationalen Gebietskörperschaften bezogen (Florian Grotz, Staatsorganisation, in: Dieter Nohlen / Florian Grotz (Hrsg.), Kleines Lexikon der Politik, 4. aktualisierte und erweiterte Auflage, München 2007, S. 542 - 545). Die kommunale Ebene bleibt dabei außen vor. 
Reform der Staatsorganisation besitzt beziehungsweise unter welchen sie eine eher geringe Rolle spielt. Auf dieser Basis werden dann die jüngeren Prozesse der Staatsreform in Deutschland, Österreich, Italien und Großbritannien näher betrachtet. Der Schlussabschnitt fasst die Untersuchungsergebnisse mit Blick auf die Ausgangsfrage zusammen.

\section{Staatsorganisation in Westeuropa: Strukturmerkmale und Entwicklungsbedingungen}

\subsection{Bundesstaaten und Einheitsstaaten: konzeptionelle Grundlagen}

Im Vergleich zu anderen Schlüsselkonzepten der politikwissenschaftlichen Institutionenlehre erweist sich die definitorische Abgrenzung von föderalen und unitarischen Staaten als ungewöhnlich schwierig. ${ }^{7}$ Während eine unübersehbar große Zahl von Föderalismusdefinitionen existiert, wird das Konzept des Einheitsstaats meist als selbstverständlich vorausgesetzt. Eine Ursache für diese definitorische Asymmetrie liegt in der Heterogenität der jeweiligen Leitprinzipien. So findet der Zentralismus seinen gleichsam natürlichen Ausdruck in der Form des Einheitsstaats, in dem die politische Souveränität ausschließlich auf nationaler Ebene angesiedelt ist. Dagegen stellt sich die staatsorganisatorische Verwirklichung des Föderalismus, das heißt die Vorstellung territorialer Einheit bei gleichzeitiger vertikaler Ausdifferenzierung ${ }^{8}$, als keineswegs eindeutig dar. Je nachdem, inwieweit man das Prinzip „Einheit in Vielfalt" (nur) an die formale Verfassungsordnung gebunden sieht oder auch als darüber hinausgehende „Lebensform“ begreift, können drei Grundverständnisse eines föderalen Systems unterschieden werden?:

- ein soziologisches Konzept, das den politischen Ausgleich territorial heterogener Interessen betont, wobei die so verstandene „föderale Gesellschaft“ mit bundesstaatlichen Organisationsstrukturen korrespondiert;

- ein konstitutionelles Verständnis, das ein föderales System an der rechtlich-institutionellen Form der Kompetenzordnung zwischen Zentralstaat und Gliedstaaten festmacht;

- eine gouvernementale Perspektive, die neben dem Verfassungsrahmen auch die politischadministrativen Akteurskonstellationen einbezieht, um das Interaktionsgefüge zwischen nationalstaatlicher Ebene und subnationalen Gebietskörperschaften zu erfassen.

Für die vorliegende Fragestellung, die sich auf institutionelle Reformen der Staatsorganisation richtet, erscheint das konstitutionelle Begriffsverständnis am besten geeignet. Will man die Kompetenzordnung in Bundes- und Einheitsstaaten kategorial unterscheiden, wird meist die Bestandsgarantie der „regionalen“ Ebene als primäres Differenzkriterium herangezogen. Demnach verfügen die regionalen Gebietskörperschaften in einem Föderalstaat in mindestens einem Politikbereich über das Letztentscheidungsrecht, wobei diese Zuständigkeit nicht einseitig von gesamtstaatlichen Einrichtungen verändert oder gar entzogen wer-

7 Ausführlich dazu Florian Grotz, Europäisierung und nationale Staatsorganisation. Institutionenpolitik in föderalen und unitarischen EU-Staaten, Baden-Baden 2006, S. 16 ff.

8 Oder in der bekannten Formulierung Elazars: „a combination of self-rule and shared rule“ (Daniel J. Elazar, Exploring Federalism, Tuscaloosa 1987).

9 Lori Thorlakson, Comparing Federal Institutions: Power and Representation in Six Federations, in: West European Politics, 26. Jg. (2003), S. $1-22$. 
den darf. Ceteris paribus haben somit gliedstaatliche Repräsentanten eine deutlich stärkere Position im Rahmen der (verfassungs-)politischen Willensbildung inne als subnationale Akteure in Einheitsstaaten.

Gleichwohl kann die Zuständigkeitsverteilung zwischen nationaler Ebene und dezentralen Gebietskörperschaften innerhalb beider Grundtypen erheblich variieren. So ist auch in unitarischen Staaten die Einrichtung von regionalen Institutionen möglich, die politischadministrative Aufgaben autonom wahrnehmen. Als weiteres Differenzkriterium wird daher der relative Umfang der den jeweiligen Ebenen zugewiesenen Kompetenzen herangezogen (Dezentralisierungsgrad). Dabei interessiert nicht nur die Verteilung legislativer und administrativer Zuständigkeiten für einzelne Politikbereiche (Aufgabenstrukturen), sondern auch die jeweiligen Gestaltungsspielräume im Rahmen der einnahmen- wie ausgabenseitigen Finanzordnung (Ressourcenstrukturen); nicht zuletzt ist von Belang, ob und inwieweit die „regionalen“ Gebietskörperschaften an der gesamtstaatlichen Willensbildung und Entscheidung beteiligt werden (Entscheidungsstrukturen). ${ }^{10}$

Auf dieser Basis lassen sich nun die unterschiedlichen Formen der Staatsorganisation typologisch ordnen (vgl. Tabelle 1). Auf der einen Seite können die Einheitsstaaten in einen „klassisch“ unitarischen und einen dezentralisierten Typ unterteilt werden; im letzteren Fall existieren auch regionale Gebietskörperschaften, die über autonome politische Kompetenzen verfügen. Auf Seite der Bundesstaaten kann ebenfalls ein stärker zentralisierter Typ, bei dem die nationale Ebene ein deutliches Übergewicht besitzt, von einem dezentral organisierten Typ unterschieden werden; zwischen diesen Ausprägungen einer „dualen“ Kompetenzordnung findet sich schließlich ein „kooperativer“ Föderalstaat, bei dem Bund und Gliedstaaten in den wichtigsten Aufgabenfeldern zusammenwirken.

\begin{tabular}{|l|l|l|l|l|l|}
\hline Tabelle 1: Typologie der Staatsorganisation \\
\hline Grundtypen & \multicolumn{3}{|c|}{ Einheitsstaat } & \multicolumn{3}{c|}{ Bundesstaat } \\
\hline Subtypen & $\begin{array}{l}\text {,klassischer" } \\
\text { Einheitsstaat }\end{array}$ & $\begin{array}{l}\text { dezentralisierter } \\
\text { Einheitsstaat }\end{array}$ & $\begin{array}{l}\text { zentralisierter } \\
\text { Bundesstaat }\end{array}$ & $\begin{array}{l}\text { kooperativer } \\
\text { Bundesstaat }\end{array}$ & $\begin{array}{l}\text { dezentralisierter } \\
\text { Bundesstaat }\end{array}$ \\
\hline $\begin{array}{l}\text { Länder- } \\
\text { beispiele }\end{array}$ & $\begin{array}{l}\text { Dänemark, } \\
\text { Griechenland, } \\
\text { Irland, } \\
\text { Portugal }\end{array}$ & $\begin{array}{l}\text { Frankreich, } \\
\text { Großbritannien, } \\
\text { Italien, } \\
\text { Spanien }\end{array}$ & Österreich & Deutschland & $\begin{array}{l}\text { Belgien, } \\
\text { Schweiz }\end{array}$ \\
\hline $\begin{array}{l}\text { a Die typologische Einordnung Spaniens erweist sich als außerordentlich schwierig, was auch in entspre- } \\
\text { chenden Bezeichnungen der Fachliteratur zum Ausdruck kommt (vgl. etwa Juan José Solozábal, Spain: } \\
\text { A Federation in the Making?, in: Joachim Jens Hesse / Vincent Wright (Hrsg.), Federalizing Europe?, } \\
\text { Oxford 1996, S. 240 - 265; Mireia Grau i Creus, Spain: Incomplete Federalism, in: Ute Wachendorfer- } \\
\text { Schmidt (Hrsg.), Federalism and Political Performance, London 2000, S. 58 - 77). Aufgrund der obigen } \\
\text { Kriterien wird das Land hier als dezentralisierter Einheitsstaat klassifiziert. } \\
\text { Quelle: Eigene Darstellung. }\end{array}$ & \\
\hline
\end{tabular}

10 Vgl. Joachim Jens Hesse / Arthur Benz, Die Modernisierung der Staatsorganisation. Institutionspolitik im internationalen Vergleich: USA, Großbritannien, Frankreich, Bundesrepublik Deutschland, Baden-Baden 1990. 
Dass diese Typologie für den westeuropäischen Kontext weiter verfeinert werden kann, dürfte unstrittig sein. ${ }^{11}$ Allerdings erscheint sie in der vorliegenden Form hinreichend geeignet, um zwei Voraussetzungen zu verdeutlichen, von denen die folgende Analyse ausgeht: Zum einen sind substanzielle Veränderungen der vertikalen Kompetenzordnung möglich, ohne dass sich damit ein Wechsel des föderalen beziehungsweise unitarischen Grundtyps verbinden muss. Auch bei Reformen der Staatsorganisation, die auf Ebene der jeweiligen Subtypen stattfinden, kann die eingangs benannte Idee der vertikalen Gewaltenteilung politisch bedeutsam werden. So ließe sich plausibel ins Feld führen, dass die demokratischen Kontrollmechanismen innerhalb einer bundesstaatlichen Ordnung verbessert würden, wenn die Gliedstaaten über mehr autonome Zuständigkeiten verfügten. Besondere Überzeugungskraft dürfte diesem Argument für jene föderalen Systeme zukommen, in denen die gesamtstaatliche Ebene eine dominante Stellung einnimmt, das heißt in kooperativen beziehungsweise zentralisierten Bundesstaaten. Zum anderen sind gewaltenteilige Zielvorstellungen, die für einen dezentral orientierten Umbau der Staatsorganisation sprechen, nicht notwendigerweise auf föderale Kontexte beschränkt. Theoretisch „passen“ sie auch als Begründung für Reformprozesse in unitarischen Staaten, etwa wenn dort die Einrichtung direkt gewählter Regionalversammlungen geplant ist, die über legislative Kompetenzen verfügen sollen.

Insgesamt erweist sich also die Idee der „vertikalen Gewaltenteilung“ in Bundes- und Einheitsstaaten als prinzipiell einschlägig, wenn es - wie in den eingangs erwähnten westeuropäischen Ländern - um eine dezentral ausgerichtete Reform der Kompetenzordnung geht. Allerdings ist damit noch nicht geklärt, ob und inwieweit die Zielvorstellung einer (verbesserten) Machtbalance zwischen nationaler Ebene und subnationalen Gebietskörperschaften tatsächlich eine institutionelle Reform anleitet. Zur Klärung dieser Frage müssen vor allem die politischen Rahmenbedingungen näher betrachtet werden, unter denen eine Veränderung der Staatsorganisation zustande kommt.

\subsection{Die Reform der Staatsorganisation: konkurrierende Zielvorstellungen und relevante Kontextbedingungen}

Unter welchen Bedingungen vollziehen sich die Reformen von föderalen und unitarischen Staaten in Westeuropa? Und welche Bedeutung kommt dabei dem Argument der „vertikalen Gewaltenteilung“ zu? Aus historischer Sicht fällt eine Antwort auf letztere Frage eher zurückhaltend aus, da die Ursprünge der Staatsorganisation in den meisten Ländern weit vor die ersten Demokratisierungsversuche zurückreichen. Wie Stein Rokkan gezeigt hat ${ }^{12}$, wurden die Grundzüge der westeuropäischen Bundes- und Einheitsstaaten wesentlich von den Prozessen der Staats- und Nationsbildung bestimmt, die im ausgehenden Mittelalter

11 Dies gilt etwa für die Berücksichtigung territorial asymmetrischer Arrangements, die nicht nur in föderalen Fällen (Regionen vs. Gemeinschaften in Belgien), sondern auch in etlichen unitarischen Staaten in jeweils unterschiedlicher Ausprägung zu finden sind (Schottland vs. Wales vs. England in Großbritannien; Grönland in Dänemark; Azoren und Madeira in Portugal). Siehe Robert Agranoff (Hrsg.), a.a.O., sowie Klaus von Beyme, Föderalismus und regionales Bewusstsein. Ein internationaler Vergleich, München 2007.

12 Stein Rokkan, Staat, Nation und Demokratie in Europa, Frankfurt am Main 2000. 
begannen. Hierbei erwiesen sich drei Strukturdimensionen der jeweiligen Territorien als besonders bedeutsam:

- die geopolitische Zugehörigkeit zum zentraleuropäischen „Städtegürtel“, der im Hochmittelalter innerhalb des Heiligen Römischen Reiches Deutscher Nation entstand und deutliche Elemente des Pluralismus und Partikularismus aufwies;

- der sozioökonomische Zentralisierungsgrad, der sich vor allem in der „mono“- beziehungsweise „polykephalen“ Struktur des Städtenetzes manifestierte; sowie

- das Ausmaß ethnisch-linguistischer Homogenität, wobei der Entwicklung einer flächendeckenden Nationalsprache ein herausragender Stellenwert zukam.

Nach Stein Rokkan war die erstbenannte Dimension insofern strukturprägend, als starke Zentren territorialer Kontrolle zunächst an den „Rändern des alten Reiches“ entstanden, die sich frühzeitig als Einheitsstaaten konsolidierten. Dazu zählten Portugal und Spanien, aber auch - in je spezifischer Weise - Frankreich, Großbritannien sowie die historischen Königreiche Nordeuropas (Dänemark, Schweden). Im Bereich des „zentralen Städtegürtels“, der sich aus ökonomisch hochentwickelten, aber politisch fragmentierten Territorien zusammensetzte, scheiterte dagegen eine militärisch-administrative Zentrumsbildung. Abgesehen von der Schweiz kam es hier zu „verspäteten“ nationalstaatlichen Einigungsprozessen. Zugleich finden sich in diesem Raum mit Deutschland, Österreich, der Schweiz, den Niederlanden (historisch) und Belgien (aktuell) alle westeuropäischen Bundesstaaten. Bis 1945 blieben die so geprägten Staatsstrukturen - von kriegsbedingten und / oder autoritären Zwischenphasen abgesehen - in ihren Grundzügen unverändert. Selbst Staaten wie Deutschland, die im 20. Jahrhundert mehrere Regimewechsel erlebten, knüpften wieder an ihre (föderative) Tradition an. ${ }^{13}$

Die hochgradige Pfadabhängigkeit ${ }^{14}$ westeuropäischer Staatsstrukturen besagt indes nicht, dass die Idee der vertikalen Gewaltenteilung politisch bedeutungslos war. Gerade im Rahmen post-autoritärer Systemwechselprozesse spielten demokratiebezogene Überlegungen bei der Ausgestaltung einer dezentralen Staatsorganisation eine beträchtliche Rolle. Dies gilt nicht nur für die Bundesrepublik Deutschland, wo bei der Erarbeitung des Grundgesetzes diverse institutionelle Lehren aus dem Scheitern der Weimarer Demokratie gezogen wurden ${ }^{15}$; ähnliche Überlegungen fanden auch in der italienischen Verfassung von 1947 und der spanischen von 1978 ihren Niederschlag. ${ }^{16}$ In der jüngeren Vergangenheit haben nun einige westeuropäische Demokratien institutionelle Reformprozesse eingeleitet,

13 Die einzige Ausnahme bildeten die Niederlande, die nach der Auflösung des Bundesstaats im Gefolge der Napoleonischen Besatzung die einheitsstaatliche Form beibehielten (während die gleichzeitig „zwangsunitarisierte“ Schweiz zu ihrer föderalen Verfassung zurückkehrte).

14 Für eine Anwendung des Pfadabhängigkeitskonzepts auf den deutschen Fall vgl. Gerhard Lehmbruch, Der unitarische Bundesstaat in Deutschland: Pfadabhängigkeit und Wandel, in: Arthur Benz / Gerhard Lehmbruch (Hrsg.), Föderalismus. Analysen in entwicklungsgeschichtlicher und vergleichender Perspektive, PVS-Sonderheft 32, Wiesbaden 2002, S. 53 - 110.

15 Siehe etwa Klaus von Beyme, Institutionelle Grundlagen der deutschen Demokratie, in: Max Kaase / Günther Schmid (Hrsg.), Eine lernende Demokratie. 50 Jahre Bundesrepublik Deutschland, WZB-Jahrbuch, Berlin 1999, S. 19 - 39.

16 Vgl. zu Italien Gaetano Sciascia, Die Verfassung der italienischen Republik vom 27. Dezember 1947 und ihre Entwicklung bis 1958, in: Jahrbuch des öffentlichen Rechts (Neue Folge), 8. Jg. (1959), S. 139 - 214; Sergio Mattarella, Das Zweikammersystem in Italien, in: Archiv des öffentlichen Rechts, 118. Jg. (1983), S. 371 - 391. Zum spanischen Fall Dieter Nohlen / Andreas Hildenbrand, Spanien, 2. Auflage, Wiesbaden 2005, S. 236 ff. 
die - in teils deutlichem Kontrast zu ihrer historischen Staats- und Verwaltungstradition auf eine umfangreiche Kompetenzverlagerung zugunsten der regionalen Ebene abzielten; hierbei könnte die Vorstellung, die demokratische Ordnung durch Dezentralisierung zu stärken, gleichfalls von Bedeutung gewesen sein.

Wie aber kann der Stellenwert des Gewaltenteilungsarguments innerhalb solcher Reformprozesse systematisch erfasst werden? Die Klärung dieses Problems erfolgt in zwei Schritten: Zunächst wird gezeigt, dass neben der vertikalen Gewaltenteilung weitere Zielvorstellungen existieren, die eine begründete Verbindung zwischen Staatsorganisation und Demokratie herstellen. Welche dieser konkurrierenden Leitideen sich im Rahmen institutionenpolitischer Reformprozesse als dominant erweist, hängt weniger von ihrer inhärenten Überzeugungskraft als vielmehr von den jeweiligen (macht-)politischen Rahmenbedingungen ab. Der zweite Schritt geht daher auf jene Kontextfaktoren ein, die das Aufkommen und die Durchsetzung bestimmter Zielvorstellungen befördern oder erschweren.

(1) Zieht man die gängige Terminologie der Systemtheorie heran, so richtet sich das Leitbild der vertikalen Gewaltenteilung auf die Input-Seite politischer Willensbildung und Entscheidung ${ }^{17}$ : Demnach soll eine bundesstaatliche Ordnung die Mehrheitsherrschaft begrenzen und so zur Stabilität und Legitimität der Demokratie beitragen. Gleichzeitig existiert eine output-orientierte Begründung für die Einführung föderativer Strukturen insofern, als von territorialer Differenzierung eine Steigerung der Wettbewerbs- und Innovationskraft einzelner Regionen und damit der Funktions- und Leistungsfähigkeit des gesamten Regierungssystems erwartet wird; diese Argumentation wird vor allem seitens der ökonomischen Föderalismustheorie vertreten. ${ }^{18}$ Mit Blick auf beide Dimensionen demokratischer Systeme finden sich auch theoretisch fundierte Positionen, die eine Zentralisierung favorisieren. Auf der Input-Seite ist dies die seit der Französischen Revolution immer wieder vorgebrachte Begründung, nach der sich die demokratische Gleichheit der Bürger am besten im Rahmen eines zentralisierten Einheitsstaats verwirklichen lässt. ${ }^{19} \mathrm{Nicht}$ zuletzt werden einer unitarischen Ordnung auch output-bezogene Vorteile zugeschrieben, da eine wirtschafts- und sozialpolitische Steuerung, wie sie etwa der Keynesianismus nahe legt, im Rahmen einheitlicher Wirtschaftsräume beziehungsweise einheitsstaatlicher Organisationsstrukturen besser bewerkstelligt werden kann als in territorial ausdifferenzierten Systemen. ${ }^{20}$ Mithin lassen sich insgesamt vier idealtypische Begründungsmuster für die (Re-) Form der Staatsorganisation anführen, die in wechselseitiger Konkurrenz zueinander stehen (vgl. Tabelle 2).

17 Zur Anwendung systemtheoretischer Begrifflichkeiten auf die Demokratietheorie grundlegend Fritz W. Scharpf, Demokratietheorie zwischen Utopie und Anpassung, Konstanz 1970.

18 Siehe Robert P. Inman / Daniel P. Rubinfeld, The Political Economy of Federalism, in: Dennis C. Mueller (Hrsg.), Perspectives on Public Choice. A Handbook, Cambridge 1997, S. 73 - 105; Wallace Oates, An Essay on Fiscal Federalism, in: Journal of Economic Literature, 37. Jg. (1999), S. 1120 - 1149; Barry Weingast, The Economic Role of Political Institutions: Market-Preserving Federalism and Economic Development, in: Journal of Law, Economics and Organization, 11. Jg. (1995), S. $1-32$.

19 Juan Linz, a.a.O., S. 396 f.

20 Vgl. Josef Esser, Keynesianismus, in: Dieter Nohlen / Florian Grotz (Hrsg.), a.a.O., S. 258 f. 


\begin{tabular}{|l|c|c|}
\hline Tabelle 2: Staatsorganisatorische Zielvorstellungen und ihr Demokratiebezug \\
\hline \multirow{2}{*}{$\begin{array}{l}\text { staatsorganisatorische } \\
\text { Zielvorstellungen }\end{array}$} & Input-Dimension & Demokratiebezug \\
\cline { 2 - 3 } & $\begin{array}{c}\text { vertikale Gewaltenteilung zur } \\
\text { dezentralisierter / } \\
\text { föderaler Staat }\end{array}$ & $\begin{array}{c}\text { erhöhte Leistungsfähigkeit durch } \\
\text { territoriale Differenzierung }\end{array}$ \\
\hline $\begin{array}{l}\text { zentralisierter / } \\
\text { unitarischer Staat }\end{array}$ & $\begin{array}{c}\text { demokratische Gleichstellung der } \\
\text { Bürger bei einheitlichem Staatsgebiet }\end{array}$ & $\begin{array}{c}\text { politische Steuerungsvorteile durch } \\
\text { unitarische Staatsorganisation }\end{array}$ \\
\hline \multicolumn{3}{|l|}{ Quelle: Eigene Darstellung. } \\
\hline
\end{tabular}

Blickt man auf die institutionenpolitischen Debatten, die in westeuropäischen Nationalstaaten seit 1945 geführt wurden, lassen sich alle benannten Positionen wiederfinden. Dabei sind durchaus bestimmte „Konjunkturen“ festzustellen. ${ }^{21}$ Unmittelbar nach dem Zweiten Weltkrieg etwa war das Zentralismusprinzip angesichts der Erfahrung mit dem Faschismus in Kontinentaleuropa weitgehend diskreditiert. Bis in die 1970er Jahre erwiesen sich dann unitarische Leitbilder im Zuge der sozialstaatlichen Entwicklung und des vorherrschenden Keynesianismus als dominant, ehe wiederum ab den 1980er Jahren - mit dem Aufkommen des Neoliberalismus und Neoregionalismus - die Vorstellung dezentraler Aufgabenwahrnehmung in der Staatsdiskussion an Bedeutung gewann.

Die zeitbedingten Moden einzelner Ideen und Argumente vermögen allerdings nicht die diversen Staatsreformen in Westeuropa seit den 1970er Jahren hinreichend zu erklären, die sich in ihrem Verlauf wie in ihrer inhaltlichen Ausrichtung erheblich voneinander unterschieden. Der wichtigste Grund dafür liegt auf der Hand: Institutionenreformen in demokratischen Regierungssystemen sind keine technokratischen Entscheidungen, sondern eminent politische Prozesse, in denen die Machtinteressen der beteiligten Akteure eine zentrale Rolle spielen. ${ }^{22}$ Von daher erscheint die Annahme naheliegend, dass im Rahmen eines institutionenpolitischen Reformprozesses mehrere gegenläufige Zielvorstellungen vertreten werden, die sich letztlich als unterschiedlich durchsetzungsfähig erweisen. Rückbezogen auf die vorliegende Problemstellung bedeutet das: Ob und inwieweit die Idee der vertikalen Gewaltenteilung bei einer Reform der Staatsorganisation handlungsleitende Funktion gewinnt, hängt primär von den jeweiligen politischen Rahmenbedingungen ab. Ihnen gilt im Folgenden das Interesse.

(2) In der empirisch-analytischen Literatur werden zahlreiche Variablen angeführt, die eine Reform der vertikalen Kompetenzordnung in föderalen und unitarischen Staaten beeinflussen. Allerdings finden sich hierzu kaum systematische Darstellungen. ${ }^{23}$ Die folgenden Ausführungen gehen daher nur auf die wichtigsten endogenen Faktoren ein und unterscheiden dazu zwischen soziostrukturellen, akteursbezogenen und institutionellen

21 Zum Folgenden ausführlich und mit weiteren Nachweisen Michiel S. de Vries, The Rise and Fall of Decentralization: A Comparative Analysis of Arguments and Practices in European Countries, in: European Journal of Political Research, 38. Jg. (2000), S. $193-224$.

$22 \mathrm{Vgl}$. André Kaiser, Mehrheitsdemokratie und Institutionenreform. Verfassungspolitischer Wandel in Australien, Großbritannien, Kanada und Neuseeland im Vergleich, Frankfurt am Main 2002.

23 Für eine neuere Bestandsaufnahme der auf Bundesstaaten bezogenen Literatur vgl. Arthur Benz, Themen, Probleme und Perspektiven der vergleichenden Föderalismusforschung, in: ders. / Gerhard Lehmbruch (Hrsg.), a.a.O., S. $9-50$. 
Erklärungsansätzen. ${ }^{24}$ Im Zentrum steht jeweils die Frage, unter welchen Rahmenbedingungen die Idee der vertikalen Gewaltenteilung eine erwartbar große Bedeutung für die Reform der Staatsorganisation besitzt beziehungsweise unter welchen sie eine eher geringe Rolle spielt.

(a) Seitens der Vergleichenden Föderalismusforschung hat der Zusammenhang zwischen bundesstaatlicher Ordnung und soziostrukturellem Kontext verstärkte Beachtung gefunden. Im Mittelpunkt steht dabei das Konzept der territorialen Konfliktlinien, das heißt das Vorhandensein einer sozioökonomisch oder soziokulturell definierten Minderheit, die auf einem Teil des Staatsgebiets konzentriert ist und von der nationalen Bevölkerungsmehrheit bestimmte Sonderrechte fordert. ${ }^{25}$ Vor diesem Hintergrund gehen zahlreiche Studien davon aus, dass eine vertikal ausdifferenzierte Staatsorganisation besonders gut zu territorial heterogenen Gesellschaften passt, und suchen festzustellen, inwieweit föderalstaatliche Arrangements die „Austarierung“ territorialer Konflikte befördern. ${ }^{26}$ In Umkehrung dieser Argumentation erscheint es auch plausibel anzunehmen, dass die gesellschaftliche Nachfrage nach (erweiterter) vertikaler Gewaltenteilung umso größer ist, je stärker die territorialen Disparitäten in einem Nationalstaat ausgeprägt sind. Ob jedoch ein derartiges Nachfragepotential zu einer Dezentralisierung der bestehenden - föderalen oder unitarischen - Staatsorganisation führt, hängt wesentlich von den am Reformprozess beteiligten Akteuren und ihren (machtpolitischen) Interessenlagen ab.

(b) Sowohl in der Föderalismus- als auch in der Regionalisierungsforschung gilt das nationale Parteiensystem als Schlüsselvariable, um die Struktur und Dynamik staatlich verfasster „Mehrebenensysteme“ zu erklären. ${ }^{27} \mathrm{Als}$ intermediäre Einrichtungen sind politische Parteien nicht nur für die Interessenvermittlung bei „normalen“ Politiken von herausragender Bedeutung, sondern auch bei Reformen der institutionellen Ordnung die entscheidenden Akteure. Darauf gründet letztlich auch die bekannte These William H. Rikers, nach der sich der (De-)Zentralisierungsgrad einer Staatsorganisation und der (De-)Zentralisierungsgrad des jeweiligen Parteiensystems wechselseitig bedingen. ${ }^{28}$ Infolgedessen liegt die Vermutung nahe, dass vertikale Gewaltenteilung umso eher als reformpolitische Zielvorstellung vertreten wird, je stärker das nationale Parteiensystem territorial ausdifferenziert ist.

24 Darüber hinaus können auch externe Faktoren die Reform der Staatsorganisation beeinflussen. Für Westeuropa ist hierbei vor allem an den supranationalen Integrationsprozess zu denken, der eine Anpassung der binnenstaatlichen Kompetenzordnung aus demokratietheoretischen Gründen nahe legt. Ausführlich dazu Tanja A. Börzel, Europäisierung und innerstaatlicher Wandel. Zentralisierung und Entparlamentarisierung, in: PVS, 41. Jg. (2000), S. 225 - 250 sowie Florian Grotz, Europäisierung und nationale Staatsorganisation, a.a.O.

25 Vgl. Ugo M. Amoretti, Introduction: Federalism and Territorial Cleavages, in: ders. I Nancy Bermeo (Hrsg.), a.a.O., S. $1-23$, S. 2.

26 Ugo M. Amoretti / Nancy Bermeo (Hrsg.), a.a.O.; Michael Burgess / John Pinder (Hrsg.), Multinational Federations, London / New York 2007.

27 Siehe unter anderem Edgar Grande, Parteiensystem und Föderalismus - institutionelle Strukturmuster und politische Dynamiken im internationalen Vergleich, in: Arthur Benz / Gerhard Lehmbruch (Hrsg.), a.a.O., S. 197 - 212; Wilfried Swenden, Federalism and Regionalism in Western Europe. A Comparative and Thematic Analysis, Basingstoke / New York 2006; Lori Thorlakson, An Institutional Explanation of Party System Congruence: Evidence from Six Federations, in: European Journal of Political Science, 46. Jg. (2007), S. 69 - 95.

28 William H. Riker, Federalism, in: FJ. Greenstein / N. Polsby (Hrsg.), Handbook of Political Science, Vol. 5: Governmental Institutions and Processes, Reading, MA 1975, S. 93 - 172, S. 137. 
Dabei erscheint es insbesondere von Belang, ob es regionalistische Parteien gibt, die bei nationalen Wahlen nur in einem Teil des Staatsgebiets antreten und dabei eine dezidiert anti-zentralistische Agenda verfolgen. ${ }^{29}$ In dieser Situation dürften nicht nur die regionalistischen Parteien selbst ihre Dezentralisierungsforderungen mit der Notwendigkeit vertikaler Machtteilung rechtfertigen; je nach der territorialen Wettbewerbssituation könnten auch landesweite Parteien von dieser Begründung elektoral profitieren und entsprechend argumentieren.

(c) Die benannten soziostrukturellen und akteursbezogenen Bedingungen, die die Relevanz der vertikalen Gewaltenteilung als reformpolitische Zielvorstellung tendenziell erhöhen, gelten gleichermaßen für unitarische und föderale Kontexte. Folgt man hingegen einem institutionellen Ansatz, werden deutliche Unterschiede zwischen beiden Grundtypen der Staatsorganisation erkennbar. Generell gilt, dass Bundesstaaten schwieriger zu reformieren sind als Einheitsstaaten, da die „regionalen“ Gebietskörperschaften in föderalen Regierungssystemen an Änderungen der vertikalen Kompetenzordnung beteiligt werden müssen, mithin eine Vetoposition innehaben. ${ }^{30}$ Diese These lässt sich insofern empirisch belegen, als „kein historisches Beispiel für den grundlegenden Umbau eines Bundesstaates unter Bedingungen demokratischer Politik“31 existiert. Unbenommen davon ist anzunehmen, dass vertikale Gewaltenteilung als Leitidee in föderalstaatlichen Reformkontexten eine tendenziell größere Rolle spielt als in einheitsstaatlichen, weil es in Bundesstaaten immer „regionale" Repräsentanten gibt, die aufgrund ihrer institutionellen Position an einer Aufwertung dezentraler Kompetenzen interessiert sind. Dazu können sie nicht nur auf mögliche Funktionsvorteile dezentralen Regierens verweisen, sondern auch und vor allem die notwendige Machtbalance zwischen Bund und Gliedstaaten ins Feld führen, die zum normativen Kern jeder föderal verfassten Demokratie gehört.

Im Fazit wird deutlich, dass die vertikale Gewaltenteilung, die in der demokratietheoretischen Diskussion einen prominenten Platz einnimmt, nicht unbedingt eine handlungsleitende Idee darstellt, wenn es um konkrete Veränderungen der Staatsorganisation geht. Reformen politischer Institutionen sind stets und in erster Linie Machtfragen. Will man also die praktische Bedeutung theoretisch begründeter Leitideen ermessen, müssen diese mit den jeweiligen Interessenkonstellationen verbunden werden. In den vorangegangenen Abschnitten wurden daher drei allgemeine Kontextbedingungen herausgearbeitet, unter denen sich vertikale Gewaltenteilung als besonders relevant für eine Staatsreform erweisen dürfte: die Existenz territorialer Konfliktlinien, ein territorial inkongruentes Parteiensystem sowie ein föderales Regierungssystem. Wie diese unterschiedlichen Variablen konkret zusammenwirken und das Auftreten bestimmter Leitideen in Prozessen der Staatsreform befördern, wird im Folgenden exemplarisch gezeigt.

29 Vgl. Lieven De Winter / Huri Türsan (Hrsg.), Regionalist Parties in Western Europe, London 1998; Wilfried Swenden, a.a.O., S. $141 \mathrm{ff}$.

30 Cheryl Saunders, The Constitutional Arrangements of Federal Systems. A Sceptical View from the Outside, in: Joachim Jens Hesse / Vincent Wright (Hrsg.), Federalizing Europe? The Costs, Benefits, and Preconditions of Federal Political Systems, Oxford 1996, S. 46 - 69, S. 49.

31 Gerhard Lehmbruch, Strategische Alternativen und Spielräume bei der Reform des Bundesstaates, in: Zeitschrift für Staats- und Europawissenschaften, 2. Jg. (2004), S. 82 - 93, S. 91. 


\section{Vertikale Gewaltenteilung im Prozess der Staatsreform: vier Fallbeispiele}

Untersucht werden vier westeuropäische Länder, die sich in der jüngeren Vergangenheit intensiv um eine Reform der vertikalen Kompetenzordnung bemüht haben: Deutschland, Österreich, Italien und Großbritannien. Diese Auswahl basiert auf einer Kombination aus Konkordanz- und Differenzmethodik (vgl. Tabelle 3). Zum einen wird mit Blick auf die soziostrukturellen, parteipolitischen und institutionellen Kontextbedingungen ein relativ breites Spektrum der in Westeuropa vorfindbaren Empirie abgedeckt. ${ }^{32}$ Zum anderen ist die Varianz insofern begrenzt, als mit den unitarischen Bundesstaaten Deutschland und Österreich und den dezentralisierten Einheitsstaaten Italien und Großbritannien relativ homogene „Zweiergruppen“ vorliegen. So sollte es auch möglich sein zu prüfen, ob das Gewaltenteilungsargument in föderalen Reformkontexten von größerer Relevanz ist als in unitarischen. Aus Umfangsgründen sind die Fallstudien eher summarisch angelegt. ${ }^{33} \mathrm{Im}$ Zentrum stehen die institutionenpolitischen Reformprozesse seit den 1990er Jahren, wobei den jeweils vertretenen Zielvorstellungen besondere Aufmerksamkeit zuteil wird.

\begin{tabular}{|l|l|l|l|l|}
\hline \multicolumn{5}{|c|}{ Tabelle 3: Die Untersuchungsfälle und ihre Kontextbedingungen (seit den 1990er Jahren) } \\
\hline & \multicolumn{1}{|c|}{ Deutschland } & \multicolumn{1}{c|}{ Österreich } & \multicolumn{1}{c|}{ Italien } & \multicolumn{1}{c|}{ Großbritannien } \\
\hline $\begin{array}{l}\text { soziostrukturelle } \\
\text { Konfliktlinien mit } \\
\text { Territorialbezug }\end{array}$ & $\begin{array}{l}\text { sozioökonomisch } \\
\text { und soziokulturell } \\
\text { bedingt homogen }\end{array}$ & $\begin{array}{l}\text { sozioökonomisch } \\
\text { und soziokulturell } \\
\text { hochgradig } \\
\text { homogen }\end{array}$ & $\begin{array}{l}\text { sozioökonomi- } \\
\text { scher / -kultureller } \\
\text { Nord-Süd- } \\
\text { Konflikt }\end{array}$ & $\begin{array}{l}\text { vor allem soziokultu- } \\
\text { reller Konflikt } \\
\text { zwischen „keltischen } \\
\text { Nationen“ und } \\
\text { Westminster }\end{array}$ \\
\hline $\begin{array}{l}\text { Struktur des } \\
\text { Parteiensystems }\end{array}$ & $\begin{array}{l}\text { konzentriertes } \\
\text { Parteiensystem } \\
\text { mit territorialen } \\
\text { Inkongruenzen }\end{array}$ & $\begin{array}{l}\text { konzentriertes, } \\
\text { weitgehend } \\
\text { kongruentes } \\
\text { Parteiensystem }\end{array}$ & $\begin{array}{l}\text { fragmentiertes, } \\
\text { bipolares } \\
\text { Parteiensystem } \\
\text { mit relevanter } \\
\text { Regionalpartei }\end{array}$ & $\begin{array}{l}\text { konzentriertes } \\
\text { Parteiensystem mit } \\
\text { relevanten Regional- } \\
\text { parteien }\end{array}$ \\
\hline $\begin{array}{l}\text { Form der } \\
\text { Staatsorganisation }\end{array}$ & Bundesstaat & Bundesstaat & Einheitsstaat & Einheitsstaat \\
\hline Quelle: Eigene Darstellung. &
\end{tabular}

\subsection{Bundesrepublik Deutschland}

Im Rahmen der föderalstaatlichen Entwicklung Deutschlands haben unitarische Leitideen seit jeher eine herausragende Rolle gespielt. ${ }^{34}$ Zwar hatten die Alliierten nach 1945 die Zielvorstellung, den westdeutschen Staat mit vertikalen checks and balances auszustatten und daher möglichst dezentral anzulegen; die bundesstaatliche Ordnung des Grundgesetzes wies jedoch von Anfang an deutlich unitarische Züge auf. ${ }^{35}$ Auch in der Folgezeit überwo-

32 Weitere für die Fragestellung interessante Fälle wären vor allem Belgien und Spanien, wo seit den 1970er Jahren zentrifugale Föderalisierungsprozesse zu beobachten sind.

33 Für detaillierte Länderanalysen siehe Florian Grotz, Europäisierung und nationale Staatsorganisation, a.a.O., S. $80 \mathrm{ff}$.

34 Vgl. Gerhard Lehmbruch, Der unitarische Bundesstaat in Deutschland, a.a.O.

35 Hans Boldt, Die Wiederaufnahme der deutschen föderativen Tradition im Parlamentarischen Rat 1948/49, in: Zeitschrift für Staats- und Europawissenschaften, 1. Jg. (2003), S. 505 - 526. 
gen gesamtstaatliche Steuerungsvorstellungen, die die Dominanz des Bundes in der Gesetzgebung festigten und zu einer erweiterten Bund-Länder-Kooperation führten; prominenteste Beispiele hierfür waren die Einführung der Gemeinschaftsaufgaben sowie der Große Steuerverbund (1967/69). Seit Mitte der 1970er Jahre wurden in der wissenschaftlichen Debatte vermehrt die Effizienz- und Effektivitätsprobleme der „Politikverflechtung“ thematisiert $^{36}$; allerdings zeitigte diese Kritik zunächst keine verfassungspolitischen Konsequenzen, da sich weder in der öffentlichen Diskussion noch unter den politischen Entscheidungsträgern eine Mehrheit für den Rückbau des „kooperativen Föderalismus“ fand.

Die Wiedervereinigung markierte dann eine nachhaltige Veränderung der Rahmenbedingungen. ${ }^{37}$ Zum einen bewirkte der Beitritt der neuen Bundesländer eine deutliche Zunahme sozioökonomischer Disparitäten und damit eine verstärkte Diversifizierung der institutionellen Eigeninteressen auf gliedstaatlicher Ebene; insbesondere seitens der reicheren westdeutschen Länder wurden nun vermehrt Forderungen nach einer Rückverlagerung legislativer und ressourcenbezogener Kompetenzen laut. Zum anderen wurde es infolge der gestiegenen Heterogenität zwischen den Parteiensystemen auf Länderebene und der stärkeren Volatilität des Wählerverhaltens zunehmend schwieriger, im Bundesrat „positive“ Mehrheiten zu formieren, was die „Störanfälligkeit“ des föderalstaatlichen Entscheidungssystems weiter verschärfte. ${ }^{38}$

Anlässlich der vereinigungsbedingten Grundgesetzreform (1992/94) wurden die benannten Trends erst in Teilen deutlich. Zwar setzten sich die stärkeren Länder bereits hier für eine kompetenzrechtliche Aufwertung der gliedstaatlichen Ebene ein, konnten aber nur sehr begrenzte Erfolge erzielen. ${ }^{39}$ Seit Ende der 1990er Jahre gewann die institutionenpolitische Debatte wieder an Intensität, wobei die Idee eines „Wettbewerbsföderalismus“ in zahlreichen Diskussionsbeiträgen als Leitidee propagiert wurde. ${ }^{40}$ Nachdem Bund und Länder im Frühsommer 2003 übereingekommen waren, eine gemeinsame „Kommission zur Modernisierung der bundesstaatlichen Ordnung“ einzusetzen, bildete dann tatsächlich die Entflechtung der vertikalen Kompetenzordnung das Hauptziel des Reformansatzes. Jedoch blieben die hierbei verfolgten Handlungsoptionen auf die Verlagerung einzelner Gesetzgebungskompetenzen auf Landesebene sowie auf den partiellen Abbau von Zustimmungsrechten des Bundesrates beschränkt. Zudem konnte hinsichtlich politisch bedeutsamer Zuständigkeiten, etwa im Bildungsbereich, kein Einvernehmen zwischen „Unitariern“ und „Föderalisten“ hergestellt werden, so dass die Kommission ohne konsensfähiges

36 Fritz W. Scharpf / Bernd Reissert / Fritz Schnabel, Politikverflechtung. Theorie und Empirie des kooperativen Föderalismus in der Bundesrepublik, Kronberg/Ts. 1976.

37 Siehe dazu ausführlich Gerhard Lehmbruch, Parteienwettbewerb im Bundesstaat. Regelsysteme und Spannungslagen im politischen System der Bundesrepublik Deutschland, 3., aktualisierte und erweiterte Auflage, Wiesbaden 2000.

38 Gerhard Lehmbruch, Strategische Alternativen, a.a.O., S. 83 f.

$39 \mathrm{Zu}$ den wichtigsten Ergebnissen in diesem Kontext zählten die grundgesetzliche Verankerung der Mitwirkung in EU-Angelegenheiten (Art. 23 GG) sowie die Anpassung der „Bedürfnisklausel“ im Rahmen der konkurrierenden Gesetzgebung (Art. 72 Abs. 2 GG). Vgl. Helge Lothar Batt, Verfassungsrecht und Verfassungswirklichkeit im vereinigten Deutschland. Die Dichotomie des Grundgesetzes zwischen limitierend-formalem und dirigierend-materialem Verfassungsverständnis, Opladen 2003.

40 Michèle Knodt, Europäisierung regionalen Regierens: Mit Sinatra zum „autonomieorientierten“ Systemwechsel im deutschen Bundesstaat, in: PVS, 43. Jg. (2002), S. 211 - 234. 
Ergebnis auseinanderging. ${ }^{41}$ Mit der unerwarteten Bildung einer Großen Koalition im Herbst 2005 kam es schließlich zur Neuauflage des Reformvorhabens und im Sommer 2006 zur Verabschiedung der formal umfangreichsten Grundgesetzänderung seit Bestehen der Bundesrepublik. Materiell ging diese „Jahrhundertreform“ jedoch kaum über den Kommissionsentwurf hinaus. So kam es lediglich zu vereinzelten Korrekturen der Politikverflechtung; eine grundlegende Neuausrichtung des kooperativen Föderalismus wurde nicht erreicht. ${ }^{42}$

Blickt man auf die konkurrierenden Leitideen, die innerhalb des skizzierten Reformprozesses vertreten wurden, ergibt sich eine deutliche Dominanz output-orientierter Begründungen. ${ }^{43}$ Dies gilt sowohl für die „Föderalisten“ (vor allem die Exekutiven der stärkeren Länder), die ihre Position mit der Kritik an der Immobilität des „überverflochtenen“ Systems und den positiven Folgewirkungen gliedstaatlichen Wettbewerbs rechtfertigten, als auch für die „Unitarier“ (insbesondere weite Teile der SPD), die eine Dezentralisierung bedeutsamer Gesetzgebungszuständigkeiten als ineffektiven „Rückfall in die Kleinstaaterei“ ablehnten. ${ }^{44}$ Daneben fand sich auch eine Position, die auf eine notwendige Stärkung der vertikalen Gewaltenteilung im deutschen Föderalismus verwies. Diese Argumentation wurde hauptsächlich von den Landesparlamenten vertreten ${ }^{45}$; den Reformprozess konnten sie aber kaum beeinflussen.

Der eher geringe Stellenwert, den das Gewaltenteilungsargument im Rahmen der deutschen Reformdebatte einnahm, ist vor allem durch institutionelle und akteursbezogene Kontextbedingungen zu erklären. Wie aufgezeigt veränderten sich auf soziostruktureller Ebene mit der Wiedervereinigung die hochgradig homogenen Voraussetzungen des kooperativen Föderalismus. Die damit einhergehenden Funktionsprobleme des verflochtenen Entscheidungssystems auf Bundesebene wurden im Verlauf der 1990er Jahre ebenso deutlich wie die strukturellen Ungleichgewichte in der Wirtschafts- und Finanzkraft zwischen den Ländern. Vor diesem Hintergrund erschien es naheliegend, die bundesstaatliche Ordnung zu entflechten. Trotz der beträchtlichen Zunahme an territorialer Heterogenität blieben die Forderungen nach gliedstaatlicher Autonomie begrenzt. Dies kann im Wesentlichen auf die unverändert unitarische Ausrichtung des politischen Wettbewerbs zurückgeführt werden: Obwohl sich die Parteiensysteme auf Landesebene während der 1990 er Jahre stärker ausdifferenzierten, gab es nach wie vor keine relevante Partei, die eine dezidiert „regionalistische Agenda“ vertrat und so auch eine erweiterte Machtteilung

41 Simon Schubert, Die „Kommission zur Modernisierung der bundesstaatlichen Ordnung“: Auftrag, Arbeitsweise, erkennbare Kompromisslinien, in: Zeitschrift für Staats- und Europawissenschaften, 3. Jg. (2005), S. $124-146$.

42 Siehe unter anderem Bernhard Vogel/ Rudolf Hrbek / Thomas Fischer (Hrsg.), Halbzeitbilanz - die Arbeitsergebnisse der deutschen Bundesstaatskommission im europäischen Vergleich, Baden-Baden 2006.

43 Florian Grotz, Europäisierung und nationale Staatsorganisation, a.a.O., S. $144 \mathrm{ff}$.

44 Beide Seiten versuchten im Übrigen auch, ihre Position durch gelegentliche Verweise auf „europäischen Anpassungsbedarf" zu untermauern; detailliert hierzu Joachim Jens Hesse / Florian Grotz, Europa professionalisieren. Kompetenzordnung und institutionelle Reform im Rahmen der Europäischen Union, Berlin 2005, S. $132 \mathrm{ff}$.

45 Uwe Thaysen, Der deutsche Föderalismus zwischen zwei Konventen, in: APuZ, B 29 - 30 (2003), S. 14 - 23; ders., Die Konventsbewegung zur Föderalismusreform in Deutschland: ein letztes Hurra der Landesparlamente zu Beginn des 21. Jahrhunderts?, in: ZParl, 35. Jg. (2004), H. 3, S. $513-539$. 
zugunsten der Länder hätte fordern können. ${ }^{46}$ Nicht zuletzt wirkten sich die Entscheidungsstrukturen des Exekutivföderalismus negativ auf die reformpolitische Bedeutung der Gewaltenteilung aus: Zwar verlangten die Landesparlamente, die aufgrund ihrer institutionellen Stellung am entschiedensten für eine föderale Machtbalance eintraten, eine angemessene Beteiligung am Reformprozess, erhielten aber erneut kein Mitentscheidungsrecht. ${ }^{47}$ Dieses blieb den Vertretern des Bundestages und des Bundesrates beziehungsweise der Landesregierungen vorbehalten.

\section{2. Österreich}

Österreich zählt zu den am stärksten zentralisierten Föderalstaaten der Welt. ${ }^{48}$ Noch mehr als in Deutschland dominiert die Bundesebene innerhalb der vertikalen Aufgaben-, Ressourcen- und Entscheidungsstrukturen, während die Länder über eine außerordentlich geringe Kompetenzausstattung verfügen. Diese gleichsam paradoxe Struktur eines „zentralisierten Bundesstaats" entstand im Gefolge des Ersten Weltkriegs, als die Republik Deutschösterreich aus der zerfallenden Donaumonarchie hervorging. ${ }^{49}$ Nach 1945 verständigten sich die rekonstituierten Länder mit der provisorischen Bundesregierung darauf, die vorfaschistische Verfassung ohne größere Diskussion wieder in Kraft zu setzen. Der ausschlaggebende Grund hierfür war, die staatliche Einheit Österreichs schnellstmöglich wiederherzustellen, wobei den alliierten Besatzungsmächten keine Gelegenheit gegeben werden sollte, auf die Verfassungsordnung Einfluss zu nehmen. Auch in der Folgezeit wurde die Entwicklung des österreichischen Bundesstaates kaum von „föderalistischen“ Zielvorstellungen geprägt. Nachdem es bis zur Wiedererlangung der völkerrechtlichen Souveränität (1955) zu weiteren Zentralisierungsmaßnahmen gekommen war, bemühten sich die Länder zwar zunehmend darum, ihre politisch-administrativen Zuständigkeiten auszubauen. Allerdings blieben die diesbezüglichen „Forderungsprogramme“, mit denen die Landeshauptleute die Bundesregierung wiederholt konfrontierten, auf kleinteilige Anpassungen beschränkt, und selbst diese bescheidene Reformagenda wurde nur in Teilen umgesetzt. ${ }^{50}$

Ende der 1980er Jahre erfuhr die österreichische Institutionenpolitik eine signifikante Veränderung. Anstelle punktueller Modifikationen sollte nun eine gesamthafte Neuordnung der binnenstaatlichen Kompetenzverteilung ausgearbeitet werden. Die sich darin

46 Während die CSU als einzige „Regionalpartei“ der alten Bundesrepublik in die Union eingebunden war und dadurch nur bedingt regionalistische Forderungen erheben konnte, blieb die PDS als „Vertreterin Ostdeutschlands“ stets pro-unitarisch ausgerichtet.

47 Florian Grotz, „Europäisierung“ der Bundesstaatsreform? Zur Übertragung des EU-Konventsmodells in Deutschland und Österreich, in: PVS, 46. Jg. (2005), S. $110-131$, S. 116 ff.

48 Ronald Watts, Comparing Federal Systems, 2. Auflage, Montreal u.a. 1999.

49 Vgl. zum Folgenden Franz Fallend, Föderalismus - eine Domäne der Exekutiven?, in: Herbert Dachs (Hrsg.), Der Bund und die Länder. Über Dominanz, Kooperation und Konflikte im österreichischen Bundesstaat, Wien u.a. 2003, S. 17 - 68; Karl Weber, Die Entwicklung des österreichischen Bundesstaates, in: Herbert Schambeck (Hrsg.), Bundesstaat und Bundesrat in Österreich, Wien 1997, S. $37-64$.

50 Siehe die detaillierte Darstellung von Heinz Pansi, Inhalt, Auswirkungen und Verwirklichung der Forderungsprogramme der Bundesländer, Wien 1992. 
dokumentierende Wende zu einem stärker systematischen Reformansatz stand in engem Zusammenhang mit dem vorgesehenen Beitritt zur Europäischen Gemeinschaft, den die Bundesregierung 1989 offiziell beantragt hatte. ${ }^{51}$ Die Länder unterstützten die EG-Mitgliedschaft nachdrücklich, befürchteten aber zugleich, dass diese sich negativ auf die föderale Gewaltenteilung auswirken würde, da ihr Zuständigkeitsbereich ohnehin stark eingeschränkt war und so bereits kleinere Kompetenztransfers auf die supranationale Ebene ihre Staatsqualität hätten in Frage stellen können. Daher verlangten die Landesregierungen nicht nur eine Beteiligung an der europapolitischen Willensbildung und Entscheidung (analog zum bundesdeutschen Modell), sondern auch eine grundlegende Reform der vertikalen Kompetenzordnung als Voraussetzung des österreichischen EG-Beitritts.

Obwohl Bund und Länder eine solche institutionelle Neuordnung im so genannten Perchtoldsdorfer Paktum (1992) vereinbarten, wurde sie weder vor dem EG-Beitritt im Jahr 1995 umgesetzt noch danach ernsthaft weiter verfolgt. ${ }^{52}$ Ende der 1990er Jahre präsentierte sich die Reformdebatte dann zweigeteilt: Während die meisten Ländervertreter weiterhin auf der Umsetzung des Paktums beharrten, kamen zunehmend Stimmen auf, die sich aus Effizienzgründen für eine „Verschlankung“ der Landesebene aussprachen. ${ }^{53}$ Auch anlässlich des jüngsten Ansatzes zu einer grundlegenden Verfassungsrevision, die der so genannte Österreich-Konvent (2003 bis 2004) erarbeiten sollte, konnte bezüglich der Föderalismusreform kein materieller Fortschritt erzielt werden. ${ }^{54}$ Zwar erörterte der Konvent unterschiedliche Modelle einer vertikalen Kompetenzordnung, zu einer (länderfreundlichen) Neuaufteilung der Gesetzgebungs-, Vollzugs- und Ressourcenzuständigkeiten kam es aber nicht. ${ }^{55}$

Im Vergleich zu Deutschland erscheinen die Zielvorstellungen, die innerhalb des österreichischen Reformprozesses vertreten wurden, stärker diffus und weniger umsetzungsorientiert. Weder wurden hier bestimmte Leitideen wie Wettbewerbsföderalismus oder Entflechtung propagiert noch die Forderungen nach einer Stärkung des Föderalismus mit konkreten Handlungsoptionen verbunden. ${ }^{56}$ Unbenommen davon lässt sich konstatieren, dass die Länder ihre Position im Wesentlichen input-orientiert begründeten, indem sie vor allem anlässlich des EG-Beitritts auf die normativ wünschenswerte (Wieder-)Herstellung

51 Ausführlich hierzu Michael Morass, Regionale Interessen auf dem Weg in die Europäische Union. Strukturelle Entwicklung und Perspektiven der Interessenvermittlung österreichischer und deutscher Landesakteure im Rahmen der Europäischen Integration, Wien 1994, S. 315 ff.

52 Theo Öhlinger, Das Scheitern der Bundesstaatsreform, in: Österreichisches Jahrbuch für Politik 1994, Wien / München 1995, S. 543 - 558.

53 Herbert Dachs, Struktur und aktuelle Fragen des Föderalismus in Österreich, in: Udo Margedant (Hrsg.), Föderalismusreform: Föderalismus in Europa I, Sankt Augustin 2002, S. 32 - 47.

54 Vgl. allgemein Österreichische Juristenkommission (Hrsg.), Der Österreich-Konvent. Eine Zwischenbilanz, Wien 2004; Peter Gerlich, The Constitutional Convention in Austria: A Parallel Campaign with Ambivalent Results, in: Zeitschrift für Staats- und Europawissenschaften, 3. Jg. (2005), S. $52-69$.

55 Peter Bußjäger, Bundesstaatsreform ja, aber welche? Modelle für die Verteilung der Gesetzgebungszuständigkeiten zwischen Bund und Ländern, in: Österreichische Zeitschrift für Politikwissenschaft, 34. Jg. (2005), S. $311-322$.

56 Siehe dazu exemplarisch die Einschätzung von Peter Pernthaler / Gert Schernthanner, Bundesstaatsreform 1994, in: Österreichisches Jahrbuch für Politik 1994, Wien / München 1995, S. 559 - 595, S. 582. 
der föderalen Gewaltenbalance verwiesen. ${ }^{57}$ Dem stand jedoch zunehmend eine Position gegenüber, die aus effizienzorientierten Gründen eine konsequentere Zentralisierung befürwortete. ${ }^{58}$

Diese Konstellation konträrer Reformvorstellungen - eine normativ begründete „Föderalisierung“ einerseits und eine funktional ausgerichtete „Zentralisierung“ andererseits - erklärt sich wiederum durch die spezifische Kombination soziostruktureller, parteipolitischer und institutioneller Kontextbedingungen. Anders als in Deutschland blieb die territoriale Homogenität der österreichischen Gesellschaft während der vergangenen Jahrzehnte weitgehend unverändert. Zumindest haben sozioökonomische oder soziokulturelle Disparitäten zwischen den Ländern die Funktionsfähigkeit des Regierungssystems nicht derart beeinträchtigt, dass sich deswegen eine Dezentralisierung der Staatsorganisation angeboten hätte. Allerdings weist der österreichische Bundesstaat seit seiner Entstehung die oben erläuterte „normative Schieflage“ zwischen föderaler Form und zentralisierter Kompetenzordnung auf. Von daher wird verständlich, dass der EG-Beitritt zum Auslöser eines umfassenderen Reformversuchs wurde, da die Europäisierung gliedstaatlicher Kompetenzen die ohnehin prekäre Machtbalance zwischen Bund und Ländern erheblich zu beeinträchtigen schien. Die bevorstehende EG-Mitgliedschaft war somit für die Landesregierungen ein willkommener Anlass, eine erweiterte föderale Gewaltenteilung zu fordern. Dass es dennoch zu keiner nennenswerten Dezentralisierung politisch-administrativer Zuständigkeiten kam, ja nicht einmal entsprechende Vorschläge unterbreitet wurden, ist auf die hochgradig unitarische Akteurskonstellation zurückzuführen: Da weder größere Inkongruenzen zwischen den Parteiensystemen auf Landesebene auftraten noch relevante Regionalparteien existierten, konnten selbst jene Bundesländer, die am ehesten „föderalistisch“ orientiert waren (Tirol, Vorarlberg), stets in die moderaten Forderungsprogramme der Ländergesamtheit eingebunden werden. ${ }^{59}$ Aus institutioneller Sicht ist nicht zuletzt zu berücksichtigen, dass die Länder auf Bundesebene über keinen wirksamen „Vetohebel“ verfügten, mit dessen Hilfe sie föderalistische Positionen hätten durchsetzen können. ${ }^{60}$ Lediglich die bevorstehende EG-Mitgliedschaft eröffnete ihnen hierzu ein Gelegenheitsfenster, da die Bundesregierung im Vorfeld des Beitrittsreferendums auf ihre politische Unterstützung angewiesen und dementsprechend zu gewissen Zugeständnissen bei der Föderalismusreform bereit war. Angesichts der fortdauernden Dominanz unitarischer Interessen auf Bundes- wie Länderebene verstrich indes auch diese außergewöhnliche Gelegenheit, die bundesstaatliche Ordnung „föderaler" auszugestalten. ${ }^{61}$

57 Michael Morass, a.a.O.

58 Vgl. unter anderem die vom Innsbrucker Institut für Föderalismus herausgegebenen „Berichte über die Lage des Föderalismus in Österreich“, Wien 1981 ff. (jährlich), insbesondere 2001, S. 9 ff.

59 Franz Fallend, a.a.O.

60 Unter den Bedingungen des österreichischen Parteiensystems kann der dortige Bundesrat nicht als effektive „Länderkammer“ funktionieren. Vgl. dazu Franz Fallend, Der Bundesrat in Österreich, in: Gisela Riescher / Sabine Ruß / Christoph M. Haas (Hrsg.), Zweite Kammern, München / Wien 2000, S. 97 - 113.

61 Jan Erk, Austria: a Federation without Federalism, in: Publius, 34. Jg. (2004), S. 1 - 20. 


\subsection{Italien}

Trotz beträchtlicher Disparitäten zwischen nördlichen und südlichen Landesteilen wurde das Königreich Italien 1861 als Einheitsstaat gegründet. ${ }^{62}$ Bis zum Ende des Zweiten Weltkriegs blieb diese unitarische Tradition ungebrochen. Die Verfassung von 1947 etablierte dann aber eine binnenstaatliche Kompetenzordnung, die die Einrichtung von Regionen mit autonomen Aufgaben- und Ressourcenkompetenzen vorsah und damit zwischen unitarischem und föderalem Strukturprinzip angesiedelt war. ${ }^{63}$ Dieser neuartige Stato regiona$1 e^{64}$ stellte einen Formelkompromiss innerhalb der Verfassunggebenden Versammlung dar, wobei weniger die normativen oder funktionalen Zielvorstellungen der beteiligten Parteien als vielmehr deren jeweilige Machtkalküle ausschlaggebend waren. ${ }^{65}$ In der Folgezeit verzögerten die regierenden Christdemokraten (DC) die Umsetzung der einschlägigen Verfassungsbestimmungen, damit die Kommunistische Partei (PCI) auch auf subnationaler Ebene von einer Machtteilhabe ausgeschlossen blieb. Dass die Regionen in den 1970er Jahren doch noch - mit allerdings stark begrenzter Kompetenzausstattung - eingerichtet wurden ${ }^{66}$, war wiederum auf veränderte Parteienkonstellationen und damit verbundene politische Tauschgeschäfte zurückzuführen; soziostrukturelle Entwicklungen und darauf bezogene Reformvorstellungen hatten erneut keine handlungsleitende Wirkung. ${ }^{67}$

Anfang der 1990er Jahre vollzog sich ein fundamentaler Wandel in der binnenstaatlichen Institutionenpolitik. ${ }^{68}$ Auslöser war zum einen die zunehmend prekäre Ineffizienz der zentralstaatlichen Umverteilung zugunsten des Mezzogiorno, die in den reichen ober-

62 Daniel Ziblatt, Structuring the State. The Formation of Italy and Germany and the Puzzle of Federalism, Princeton 2006.

63 Vgl. Gaetano Sciascia, a.a.O.

64 Zwar fand sich in der spanischen Verfassung von 1931 ein ähnlich strukturierter Regionalstaat, doch war diese nur von kurzer Dauer.

65 Gemäß ihrer ideologischen Ausrichtung präferierte die Christdemokratie ursprünglich eine dezentralisierte Staatsorganisation, sprach sich aber bei den Verfassungsverhandlungen dagegen aus, um die absehbare Regierungsbeteiligung der Kommunistischen Partei in den mittelitalienischen Regionen zu verhindern. Die Position des PCI war genau umgekehrt: Obwohl gemäß dem „demokratischen Zentralismus" eindeutig unitarischen Leitideen verpflichtet, befürwortete die Partei ähnlich wie auch die Sozialisten (PSI) - aus machtpolitischen Gründen die Schaffung einer Regionalebene. Unbenommen davon erwiesen sich die Optionen für die Ausgestaltung der Staatsorganisation als begrenzt, da angesichts diverser separatistischer Tendenzen eine stark föderalistische Ordnung ebenso ausschied wie eine nahtlose Rückkehr zum „klassischen“ Einheitsstaat. Ausführlich dazu Alexander Grasse, Italiens langer Weg in den Regionalstaat. Die Entstehung einer Staatsform im Spannungsfeld von Zentralismus und Föderalismus, Opladen 2000, S. 165 ff.

66 Dies bezieht sich nur auf die 15 Regionen „mit Normalstatut“; die fünf Regionen „mit Sonderstatut" wurden bereits zuvor institutionalisiert.

67 So wurden die entsprechenden Reformschritte wesentlich dadurch befördert, dass der PSI in den 1960er Jahren seine Beteiligung an der DC-Regierung von der Einrichtung von Regionalorganen abhängig machte. Auch die Dezentralisierungsmaßnahmen in den 1970er Jahren kamen vor allem zustande, weil die christdemokratischen (Minderheits-)Kabinette teilweise auf die Unterstützung der sozialistischen beziehungsweise kommunistischen Parlamentsfraktionen angewiesen waren. Siehe dazu ausführlich Robert Leonardi, The Regional Reform in Italy: From Centralized to Regionalized State, in: ders. (Hrsg.), The Regions and the European Community, London 1993, S. 217 - 246, S. $222 \mathrm{ff}$.

68 Florian Grotz, Europäisierung und nationale Staatsorganisation, a.a.O., S. 239 ff. 
italienischen Regionen den Aufstieg regionalistischer Parteien - allen voran der Lega Nord (LN) - beförderte. Zum anderen bot der fast zeitgleiche Zusammenbruch des traditionellen Parteiensystems auch und gerade der LN die Möglichkeit, sich mit ihrer anti-zentralistischen Programmatik innerhalb des neuen Wettbewerbsspektrums zu positionieren. Erstmals in der italienischen Nachkriegsgeschichte entwickelte sich so eine Föderalismusdebatte, an der alle relevanten Parteien mehr oder minder aktiv beteiligt waren. Zudem lancierten rechte wie linke Regierungsbündnisse seit Mitte der 1990er Jahre diverse Dezentralisierungsinitiativen. ${ }^{69}$ Der wohl wichtigste Schritt in diesem Prozess war eine konstitutionelle Neuordnung der legislativen Gesetzgebungszuständigkeiten zugunsten der Regionen (Titel V der Italienischen Verfassung), die 2001 von der Linksregierung unter Massimo D’Alema gegen die Stimmen der rechten Opposition verabschiedet und per Referendum bestätigt wurde. ${ }^{70}$ Die von 2001 bis 2006 amtierende Rechtskoalition, an der auch die LN beteiligt war, verzögerte nicht nur die Umsetzung der „linken“ Verfassungsänderungen, sondern erarbeitete auch einen eigenen Entwurf für eine erweiterte Dezentralisierung („devoluzione“), den sie gleichfalls gegen den Widerstand der parlamentarischen Opposition durchsetzte. ${ }^{71}$ Diese Vorlage wurde schließlich am 25. Juni 2006 von einer deutlichen Wählermehrheit in einem Referendum zurückgewiesen. ${ }^{72}$ Die Reform der italienischen Staatsorganisation kann damit noch nicht als abgeschlossen gelten. ${ }^{73}$ Als Zwischenergebnis bleibt festzuhalten, dass die Regionen während der vergangenen 15 Jahre deutlich erweiterte Kompetenzen vor allem im legislativen und administrativen Bereich erhielten; von einer bundesstaatlichen Ordnung ist Italien allerdings nach wie vor deutlich entfernt. ${ }^{74}$

Dieser ebenso komplexe wie dynamische Reformprozess kann hier nicht im Einzelnen nachgezeichnet werden. Insgesamt ist zu konstatieren, dass in den Verfassungsdebatten um eine „Föderalisierung“ der Staatsorganisation output-orientierte Zielvorstellungen überwogen. ${ }^{75}$ Dies trifft auch für die Lega Nord zu, die bisweilen auf kulturelle Differenzen zwischen Nord- und Süditalien verwies, jedoch den Hauptakzent ihrer föderalistischen beziehungsweise separatistischen Kampagnen auf das wirtschafts- und finanzpolitische

69 Ausführlich hierzu und zum Folgenden Anna Cento Bull / Mark Gilbert, The Lega Nord and the Northern Question in Italian Politics, Houndmills u.a. 2001; Thomas W. Gold, The Lega Nord and Contemporary Politics in Italy, Houndmills u.a. 2003; Margarita Gómez-Reino Cachafeiro, Ethnicity and Nationalism in Italian Politics: Inventing the Padania, Lega Nord and the Northern Question, Aldershot u.a. 2002.

70 Maurizio Cotta / Luca Verzichelli, Political Institutions in Italy, Oxford 2007, S. 190 ff.

71 Vgl. Alexander Grasse, a.a.O., S. $370 \mathrm{ff}$.

72 Bei einer Wahlbeteiligung von 53,7 Prozent lehnten 61,7 Prozent der Abstimmenden die Verfassungsänderung ab (Daten nach Research Centre on Direct Democracy, www.c2d.ch).

73 Maurizio Cotta / Luca Verzichelli, a.a.O., S. 194 f.

74 In der italienischen Diskussion wird die gegenwärtige Kompetenzordnung zwar häufig als „kooperativer Föderalismus" bezeichnet, ist aber nach oben definierten Kriterien unverändert als dezentralisierter Einheitsstaat zu klassifizieren.

75 Vgl. hierzu unter anderem Jens Woelk / Francesco Palermo, Italien auf dem Weg zum Bundesstaat? Ein Überblick über den Reformprozess, in: Jahrbuch für Italienisches Recht, 11. Jg. (1998), S. 185 - 204; Jens Woelk, Föderalismus per Dekret? Zum Stand der Bassanini-Reformen in den Beziehungen Staat und Regionen, in: Jahrbuch für Italienisches Recht, 13. Jg. (2000), 105 - 124; sowie die kontinuierlichen Länderberichte in: Europäisches Zentrum für Föderalismusforschung (Hrsg.), Jahrbuch des Föderalismus, Baden-Baden, 2000 ff. (jährlich). 
„Versagen“ des Zentralstaats legte. ${ }^{76}$ Auch einfachgesetzliche Dezentralisierungsmaßnahmen, die vor allem unter der Regierung von Romano Prodi (1996 bis 1998) durchgeführt wurden, waren nicht dem Ziel einer vertikalen Gewaltenteilung verpflichtet, sondern dienten vor allem dem Zweck, die budgetäre Konsolidierung des Gesamtstaats unter Einbezug der Regionen voranzutreiben, um den Beitritt Italiens zur dritten Stufe der Europäischen Wirtschafts- und Währungsunion sicherzustellen. ${ }^{77}$

Im Fazit kann die Dominanz output-bezogener Begründungen für die Reform der italienischen Staatsorganisation mit den krisenhaften Veränderungen der sozioökonomischen Rahmenbedingungen und - damit einhergehend - der Neuordnung des parteipolitischen Wettbewerbs seit den 1990er Jahren erklärt werden. Vor dem Hintergrund des „Staatsversagens" wurde Dezentralisierung vor allem als funktionale Lösung angesehen, um die Effektivität und damit auch Legitimität des politischen Systems zu stabilisieren beziehungsweise zu erhöhen. Die normative Leitidee der vertikalen Gewaltenteilung spielte hierbei keine erkennbare Rolle, zumal die meisten Parteien beider politischer Lager letztlich in ihrer Programmatik unitarisch ausgerichtet blieben. ${ }^{78}$ Radikal föderalistische Positionen, wie sie die LN vertrat, fanden zwar temporär Anklang in der norditalienischen Wählerschaft, waren aber nicht dauerhaft mehrheitsfähig. ${ }^{79}$ Schließlich wirkte auch das einheitsstaatliche Regierungssystem einer politischen Bedeutung der vertikalen Gewaltenteilung tendenziell entgegen: Zwar gewannen die Vertreter der italienischen Regionaleinrichtungen im Zuge des Reformprozesses an politischer Sichtbarkeit und verschafften dadurch ihren institutionellen Eigeninteressen deutlicher Gehör ${ }^{80}$; anders als die deutschen und österreichischen Länder verfügten sie aber nicht über die institutionelle Legitimitätsressource, die eine bereits bestehende bundesstaatliche Ordnung für das Argument der föderalen Machtbalance darstellt.

\subsection{Großbritannien}

Im internationalen Vergleich ist die Staatsorganisation Großbritanniens $s^{81}$ durch eine sehr spezifische Ausgangssituation gekennzeichnet. ${ }^{82}$ Nachdem die englische Krone bis Anfang des 18. Jahrhunderts die gesamte Insel unter ihre Kontrolle gebracht hatte, formierte sich

76 John Agnew, The Rhetoric of Regionalism: The Northern League in Italian Politics, 1983-94, in: Transactions of the Institute of British Geographers, 20. Jg. (1995), S. 156 - 172.

77 Sergio Fabbrini / Marco Brunazzo, Federalizing Italy: The Convergent Effects of Europeanization and Domestic Mobilization, in: Regional and Federal Studies, 13. Jg. (2003), S. $100-120$.

78 Florian Grotz, Europäisierung und nationale Staatsorganisation, a.a.O., S. 258 f.

79 Vgl. M. Chiara Barlucchi / Volker Dreier, Der Schlaf der Politik gebiert ein Ungeheuer. Zu den Sezessionsbestrebungen der Lega Nord, ihren Ursachen und möglichen Erfolgsaussichten, in: ZPol, 8. Jg. (1998), S. 569 - 596, S. 582 ff.; Thomas W. Gold, a.a.O., S. 105.

80 Carlo Desideri / Vincenzo Santantonio, Building a Third Level in Europe: Prospects and Difficulties in Italy, in: Charlie Jeffery (Hrsg.), The Regional Dimension of the European Union. Towards a Third Level in Europe?, London 1997, S. 96 - 116; Maurizio Cotta / Luca Verzichelli, a.a.O.

81 Die Sonderstellung Nordirlands im Rahmen des Vereinigten Königreichs wird hier nicht eigens berücksichtigt.

82 Vgl. aus der umfangreichen Literatur repräsentativ Derek W. Urwin, Territorial Structures and Political Developments in the United Kingdom, in: Stein Rokkan / Derek Urwin (Hrsg.), The Politics of Territorial Identity: Studies in European Regionalism, London 1982, S. 19 - 73; Vernon Bogdanor, Devolution, Oxford 2001. 
das britische Gemeinwesen als zentralistisch strukturierter Einheitsstaat. Gleichwohl wurden die anderen Nationen, Waliser und Schotten, nicht vollständig assimiliert, sondern durften jeweils bestimmte Sonderrechte im kulturellen Bereich oder im Gerichtswesen (Schottland) beibehalten. Politisch-administrativ wurde das gesamte Territorium der Gesetzgebung Westminsters unterstellt. Da die binnenstaatliche Entwicklung Großbritanniens im weiteren Verlauf keine größeren Brüche mehr verzeichnete, blieb die Parlamentssouveränität als das staatsorganisatorische Leitprinzip unverändert erhalten. Dies schloss nicht aus, dass eigene Ministerien für Schottland und Wales eingerichtet und im Parlament gesonderte Regionalausschüsse gebildet wurden. Eine Dezentralisierung politisch-administrativer Kompetenzen erschien dagegen nicht nur mit dem Westminsterprinzip unvereinbar, sondern wurde auch nach 1945 von keiner der beiden großen Parteien ernsthaft in Erwägung gezogen. ${ }^{83}$

Ende der 1960er Jahre veränderten sich die innenpolitischen Rahmenbedingungen insofern, als im Zuge des sich verstärkenden „keltischen Nationalismus“ regionalistische Parteien in Schottland (Scottish National Party, SNP) und Wales (Plaid Cymru) wiederholt Wahlerfolge erzielen konnten. ${ }^{84}$ Dies nahmen die Labour-Regierungen unter Harold Wilson und James Callaghan zum Anlass, eine Übertragung politisch-administrativer Kompetenzen auf direkt gewählte Versammlungen in den beiden nicht-englischen Regionen vorzusehen. ${ }^{85}$ Mit der so genannten devolution verband sich indes keine Abkehr von der unitarischen Staatstradition; vielmehr sollte dadurch die politische und wirtschaftliche Einheit des Königreichs gestärkt werden, zumal das Westminsterparlament die uneingeschränkte Kompetenz-Kompetenz behalten würde. ${ }^{86}$ Gleichwohl traf die Initiative auf den entschiedenen Widerstand nicht nur der konservativen Opposition, sondern auch von Teilen der LabourFraktion. Vor diesem Hintergrund konnten die Devolution Acts (1978) auch nicht der Bevölkerungsmehrheit vermittelt werden: Entsprechende Referenden, die in Schottland und Wales im März 1979 stattfanden, verfehlten jeweils die vorgegebenen Quoren. ${ }^{87}$

Während der konservativen Regierungsära (1979 bis 1997) wurde kein weiterer Dezentralisierungsversuch unternommen. ${ }^{88}$ Allerdings stellte sich Labour, die auch während dieser langen Oppositionsphase stärkste politische Kraft in beiden „keltischen“ Landesteilen blieb, an die Spitze entsprechender Reformbestrebungen. Schließlich nahm die Partei auch einen devolution-Entwurf in ihr Wahlprogramm auf, der in weiten Teilen den Gesetzen

83 Siehe die detaillierte Darstellung bei Michael Münter, Verfassungsreform im Einheitsstaat. Die Politik der Dezentralisierung in Großbritannien, Wiesbaden 2005.

84 Ausführlich zum Hintergrund Roland Sturm, Nationalismus in Schottland und Wales, 1966-1980. Eine Analyse seiner Ursachen und Konsequenzen, Bochum 1981, S. $151 \mathrm{ff}$.

85 Vgl. zum Folgenden Dietmar Ehm, Devolution in Schottland und Wales. Ethnonationale Vielfalt, Problembereiche staatlicher Entwicklung und territoriale Gestaltungselemente britischer Politik, München 1988, S. 311 ff.; Peter Malanczuk, Region und unitarische Struktur in Großbritannien. Die verfassungsrechtliche und verwaltungsorganisatorische Bedeutung der Region in England, Schottland und Wales, Berlin u.a. 1984, S. $181 \mathrm{ff}$.; Michael Münter, a.a.O., S. $147 \mathrm{ff}$.

86 Siehe dazu beispielhaft die Ausführungen des Weißbuchs „Democracy and Devolution. Proposals for Scotland and Wales“, London, Cmnd. 5732, das die Regierung Wilson 1974 vorlegte.

87 Denis Balsom / Ian McAllister, The Scottish and Welsh Devolution Referenda of 1979: Constitutional Change and Popular Choice, in: Parliamentary Affairs, 32. Jg. (1979), S. $394-409$.

88 Zum Folgenden Florian Grotz, Europäisierung und nationale Staatsorganisation, a.a.O., S. $297 \mathrm{ff}$. 
von 1978 glich. Nach dem Mehrheitswechsel 1997 setzte die Regierung Tony Blairs die geplante Dezentralisierung zügig um. Dabei wurden dem schottischen Parlament relativ weitreichende legislative Zuständigkeiten zugewiesen, während die walisische Versammlung nur administrative Kompetenzen erhielt und in England zunächst überhaupt keine regionalen Institutionen gebildet wurden. Damit war der asymmetrische devolution-Prozess noch nicht beendet; allerdings blieb er von regional unterschiedlichen Reformforderungen und entsprechend differenzierten Reaktionen der Zentralregierung bestimmt: Während die walisische Versammlung einen Ausbau ihrer Kompetenzausstattung analog zu Schottland verlangte, wurde die Schaffung einer gewählten Regionalversammlung in Nordostengland 2004 von der Bevölkerung per Referendum klar zurückgewiesen. Infolgedessen stellte Whitehall auch die Pläne einer englandbezogenen devolution bis auf weiteres ein.

Hinsichtlich der Zielvorstellungen, die sich mit dem skizzierten Reformprozess verbanden, fällt ein grundlegendes Spannungsverhältnis auf: Einerseits führte die Labour-Regierung umfangreiche Dezentralisierungsmaßnahmen durch, andererseits stellte sie das unitarische Westminsterprinzip zu keinem Zeitpunkt zur Disposition. Von Anfang an basierte die devolution weder auf der Vorstellung einer föderalen Balance noch auf output-orientierten Erwartungen im Sinne der ökonomischen Föderalismustheorie. ${ }^{89}$ Ausschlaggebend waren vielmehr machtpolitische Kalküle: So ging Labour auf die jeweiligen Autonomieforderungen in ihren schottischen und walisischen Hochburgen nur so weit wie nötig ein, um der Konkurrenz durch SNP und Plaid Cymru offensiv zu begegnen. ${ }^{90}$ Trotz der Existenz territorialer Konfliktlinien und regionalistischer Parteien war die Idee der vertikalen Gewaltenteilung also auch im britischen Fall nicht handlungsleitend, da beide großen Parteien sowohl Konservative als auch Labour - an ihrer zentralistischen Staatsvorstellung festhielten. Dass die Blair-Regierung diese unitarische Grundhaltung trotz ihrer Dezentralisierungspolitik beibehalten konnte, wurde nicht zuletzt durch eine institutionelle Besonderheit des britischen Regierungssystems ermöglicht: Wäre das staatsorganisatorische Leitprinzip der Parlamentssouveränität in einer geschriebenen Verfassung verankert gewesen, hätte es in Anbetracht der umfangreichen Dezentralisierung wahrscheinlich verändert oder zumindest anders definiert werden müssen. So aber konnte Labour unschwer an der Behauptung festhalten, das Westminstersystem werde durch die devolution in keiner Weise beeinträchtigt. ${ }^{91}$

89 Zwar verband die Labour-Regierung die Dezentralisierung gelegentlich mit einer verbesserten Funktions- und Leistungsfähigkeit des politischen Systems, doch wurde diese Argumentation nie wirklich konkret. Exemplarisch sei auf das Weißbuch für Wales von 1997 verwiesen, in dem betont wurde, dass die Einrichtung einer Regionalversammlung wirtschaftliche Vorteile für Wales als ,erfolgreiche europäische Region“ bringen würde. Allerdings wurde diese Begründung nicht erläutert, geschweige denn mit dem institutionellen Reformvorhaben verknüpft.

90 Siehe dazu auch Roland Sturm, Devolutions- und Verfassungsreformprozesse in Großbritannien, in: Udo Margedant (Hrsg.), Föderalismusreform: Föderalismus in Europa II, Sankt Augustin 2002, S. $48-63$.

91 James Mitchell, Territorial Politics and Change in Britain, in: Peter Catterall / Wolfram Kaiser / Ulrike Walton-Jordan (Hrsg.), Reforming the Constitution. Debates in Twentieth-Century Britain, London u.a. 2000, S. 225 - 254. 


\section{Im Vergleich: vertikale Gewaltenteilung im Reformprozess}

Die vorangegangenen Fallstudien haben gezeigt, dass der länderübergreifende Trend zu dezentralen Staatsstrukturen in Westeuropa nicht monokausal zu erklären ist, sondern auf kontextspezifischen Konstellationen unterschiedlicher Variablen beruht. Dabei spielte die normative Zielvorstellung, die vertikale Gewaltenteilung zwischen den politisch-administrativen Ebenen zu verbessern, eine nur untergeordnete Rolle. Einzig in Österreich kam dem Argument der föderalen Machtbalance eine gewichtige Bedeutung für die intendierte Neuordnung des zentralistischen Bundesstaats zu; eine entsprechende Reform steht allerdings bis heute aus. In Deutschland vertraten lediglich die Landesparlamente eine ähnlich begründete Position, während die Dezentralisierungsprozesse in Italien und Großbritannien durch andere Argumentationsmuster und Motive geprägt waren. Sowohl der allgemeine Befund zum Stellenwert der vertikalen Gewaltenteilung in den Reformprozessen als auch die länderbezogenen Spezifika sind auf die jeweiligen soziostrukturellen, parteipolitischen und institutionellen Rahmenbedingungen zurückzuführen.

Blickt man zunächst auf die soziostrukturellen Kontexte, werden einige Zusammenhänge mit den vorherrschenden Reformbegründungen erkennbar. Sowohl in Deutschland als auch in Italien kam es Anfang der 1990er Jahre zu einer nachhaltigen Verschärfung beziehungsweise Politisierung territorialer Disparitäten, was die Funktions- und Leistungsfähigkeit der Staatsorganisation erkennbar beeinträchtigte. Mithin dominierten output-orientierte Argumente die jeweiligen Reformdebatten über eine Föderalisierung des Zentralstaats (Italien) beziehungsweise eine Entflechtung des unitarischen Föderalismus (Deutschland). In Großbritannien hingegen stellte die gesellschaftliche Nachfrage nach regionaler Autonomie in Schottland und Wales, die sich bereits seit Anfang der 1970er Jahre in wachsender Unterstützung für regionalistische Parteien dokumentierte, eine genuin politische Herausforderung für die Labour Party dar, die traditionell in beiden „keltischen“ Landesteilen dominierte; output-orientierte Erwägungen waren in diesem Kontext ebenso wenig handlungsleitend wie das normative Ziel einer vertikalen Gewaltenteilung. ${ }^{92}$ In Österreich schließlich blieb die territorial homogene Sozialstruktur weitgehend unverändert. Das Hauptproblem der bundesstaatlichen Ordnung lag daher nicht in einer verschlechterten Output-Bilanz, sondern in dem normativ prekären Ungleichgewicht zwischen föderaler Form und zentralisierter Kompetenzordnung. So konnte der bevorstehende EG-Beitritt zum Auslöser eines Reformprozesses werden, bei dem die Länder die Neuordnung der binnenstaatlichen Kompetenzverteilung mit der Aufrechterhaltung der vertikalen Gewaltenbalance im europäischen Rahmen - allerdings ohne Erfolg - begründeten.

Inwieweit die veränderten gesellschaftlichen Rahmenbedingungen tatsächlich institutionellen Reformbedarf erzeugten und in welche Handlungsoptionen dieser übersetzt wurde, hing wesentlich von parteipolitischen Akteurskonstellationen ab. Wie aufgezeigt stellte sich die soziostrukturelle Ausgangssituation in Deutschland und Österreich durchaus unter-

92 Zweifellos wurde die anti-zentralistische Haltung in der „keltischen Peripherie“ auch durch bestimmte Maßnahmen der Londoner Regierung - vor allem in der Ära Thatcher - gestärkt. Gleichwohl sind die devolution-Forderungen in Schottland und Wales nicht primär mit territorial bezogenen Performanzproblemen der unitarischen Staatsorganisation zu erklären, zumal insbesondere Schottland von der bestehenden zentralstaatlichen Finanzordnung überproportional profitierte. Ausführlich dazu Michael Münter, a.a.O. 
schiedlich dar. Die unitarische Wettbewerbsstruktur des nationalen Parteiensystems sorgte jedoch jeweils dafür, dass prononciert föderalistische Positionen im Reformprozess keine qualifizierten Mehrheiten fanden. Demgegenüber wiesen die Parteiensysteme Italiens wie Großbritanniens stärkere territoriale Inkongruenzen auf. In beiden Fällen waren regionalistische Parteien die wichtigsten Triebkräfte der anti-zentralistischen Reformen, nahmen dabei aber unterschiedliche Rollen ein. So konnte die Lega Nord angesichts der italienischen Systemkrise nicht nur eine breite Föderalismusdebatte anstoßen, sondern wirkte auch in der Regierung an der Ausarbeitung einzelner Reformentwürfe mit. Der Einfluss der schottischen und walisischen Nationalisten auf den britischen devolution-Prozess war eher indirekter Natur: Durch ihre Wahlerfolge wurde die regierende Labour Party dazu veranlasst, entgegen ihrer unitarischen Programmatik eine Dezentralisierung des Vereinigten Königreichs anzugehen. Unbenommen davon verwies keine der benannten regionalistischen Parteien auf das Gewaltenteilungsargument, um ihre territorialen Autonomieforderungen zu begründen.

Weitaus größeren Einfluss darauf, ob die vertikale Machtbalance als Zielvorstellung innerhalb eines Reformprozesses präsent war, hatte offenbar die institutionelle Struktur der Staatsorganisation. Nicht zufällig wurde dieses Argument in beiden föderalen Fällen von jenen Akteuren am nachdrücklichsten vertreten, deren raison d'être von Unitarisierung und Europäisierung am stärksten bedroht zu sein schien. Im deutschen Exekutivföderalismus waren und sind dies die Landesparlamente, während im zentralistischen Bundesstaat Österreich tendenziell die gesamte Landesebene betroffen war und entsprechend argumentierte. In den Einheitsstaaten Italien und Großbritannien hingegen machten die Vertreter der Regionaleinrichtungen von der vertikalen Machtbalance als institutionenpolitischer Leitidee bislang keinen vergleichbaren Gebrauch. Dies stützt tendenziell den institutionellen Ansatz, nach dem das Gewaltenteilungsargument in föderalen Reformkontexten einen größeren Stellenwert besitzt als in unitarischen.

Die (demokratie-)theoretischen Argumente über Vor- und Nachteile von bundes- und einheitsstaatlichen Systemen finden sich also nur sehr partiell in jenen Reformprozessen wieder, die etliche westeuropäische Länder in der jüngeren Vergangenheit durchlaufen haben. Aus normativer Sicht mag dieser Befund ernüchternd anmuten. Allerdings könnte er auch als Ansporn dienen, theorieorientierte Staatslehre und Vergleichende Systemforschung stärker aufeinander zu beziehen. Dadurch würde nicht nur das Wissen über die Kontextbedingtheit institutionenpolitischer Zielvorstellungen erweitert; vielmehr erhielte auch die Diskussion über Gewaltenteilung im demokratischen Staat eine zusätzliche empirische Grundierung, derer sie offenbar dringend bedarf, um auch in der Politikwissenschaft wieder stärker wahrgenommen zu werden. ${ }^{93}$

93 Siehe dazu Everhard Holtmann, Dynamische Gewaltenteilung - ein „vergessenes“ Thema in der Politikwissenschaft, in: PVS, 45. Jg. (2004), S. $311-320$. 